\title{
Lowering blood cholesterol does not affect neuroinflammation in experimental autoimmune encephalomyelitis
}

\section{solenne vigne}

CHUV: Centre Hospitalier Universitaire Vaudois https://orcid.org/0000-0002-8786-0198

\section{Donovan Duc}

CHUV: Centre Hospitalier Universitaire Vaudois

\section{Yannick Yersin}

CHUV: Centre Hospitalier Universitaire Vaudois

\section{Jessica Rebeaud}

CHUV: Centre Hospitalier Universitaire Vaudois

\section{Florian Ruiz}

CHUV: Centre Hospitalier Universitaire Vaudois

\section{Benjamin Peter}

CHUV: Centre Hospitalier Universitaire Vaudois

\section{Tinh-Hai Collet}

Geneva University Hospitals: Hopitaux Universitaires Geneve

\section{Caroline Pot ( $\sim$ Caroline.Pot-kreis@chuv.ch )}

Geneva University Hospitals: Hopitaux Universitaires Geneve https://orcid.org/0000-0002-1146-3129

\section{Research}

Keywords: Autoimmunity, Multiple sclerosis, EAE, neuroinflammation, cholesterol, familial hypercholesterolemia, LDL receptor, PCSK9 monoclonal antibody

Posted Date: March 17th, 2021

DOl: https://doi.org/10.21203/rs.3.rs-315230/v1

License: (c) (i) This work is licensed under a Creative Commons Attribution 4.0 International License. Read Full License

Version of Record: A version of this preprint was published at Journal of Neuroinflammation on February 7th, 2022. See the published version at https://doi.org/10.1186/s12974-022-02409-x. 
1 Lowering blood cholesterol does not affect neuroinflammation in experimental 2 autoimmune encephalomyelitis

3

4 Solenne Vigne ${ }^{1 *}$, Donovan Duc ${ }^{1 *}$, Yannick Yersin ${ }^{1}$, Jessica Rebeaud ${ }^{1}$, Florian Ruiz ${ }^{1}$, Benjamin

$5 \quad$ Peter $^{1}$, Tinh-Hai Collet ${ }^{2}$, Caroline Pot $^{1}$

$6 *$ equal contribution

7

8

9

1. Laboratories of Neuroimmunology, Neuroscience Research Center and Service of Neurology, Department of Clinical Neurosciences, Lausanne University Hospital, Chemin des Boveresses 155, 1066 Epalinges, Lausanne, Switzerland.

2. Service of Endocrinology, Diabetes, Nutrition and Therapeutic Education, Department of Medicine, Geneva University Hospitals (HUG), Rue Gabrielle-Perret-Gentil 4, 1211 Geneva 14, Switzerland.

Lead contact information and corresponding author: Caroline Pot, Department of Clinical Neurosciences, Lausanne University Hospital, 1011 Lausanne, Switzerland; email: Caroline.Pot-kreis@chuv.ch; ORCID: 0000-0002-1146-3129 


\section{Abstract}

Background: Multiple sclerosis (MS) is a chronic disabling disease of the central nervous system (CNS) commonly affecting young adults. There is increasing evidence that environmental factors are important in the development and course of MS. Metabolic syndrome (MetS) including dyslipidemia has been associated with a worse outcome in MS disease. Furthermore, the lipid lowering drugs statins have been proposed to improve MS disease course. However, cholesterol is also rate-limiting for myelin biogenesis and promotes remyelination in MS animal models. Thus, the impact of circulating blood cholesterol level during the disease remains debated and controversial.

Methods: We assessed the role of circulating cholesterol on the murine model of MS, the experimental autoimmune encephalomyelitis (EAE) disease using two different approaches: 1) the mouse model of familial hypercholesterolemia induced by low density lipoprotein receptor (LDLr) deficiency, and 2) the use of the monoclonal anti-PCSK9 neutralizing antibody alirocumab which reduces LDLr degradation and consequently lowers blood levels of cholesterol.

Results: Elevated blood cholesterol levels induced by LDLr deficiency did not worsen clinical symptoms of mice during EAE. In addition, we observed that the anti-PCSK9 antibody alirocumab did not influence EAE disease course, nor modulate the immune response in EAE.

Conclusions: These findings suggest that blood cholesterol level has no direct role in neuroinflammatory diseases and that the previously shown protective effects of statins in MS are not related to circulating cholesterol.

Keywords: Autoimmunity, Multiple sclerosis, EAE, neuroinflammation, cholesterol, familial hypercholesterolemia, LDL receptor, PCSK9 monoclonal antibody. 


\section{Background}

Multiple sclerosis (MS) is a chronic inflammatory and autoimmune disease affecting the central nervous system (CNS) leading to neuronal damage and disabling neurological deficits (1). It is a common disorder affecting young adults; its mortality is low but it is a lifelong disease with high morbidity. The etiology of MS is multifactorial and environmental factors play a major role in disease causation (2). In line with this concept, obesity is associated with increased risk of MS $(3,4)$ and several studies have shown that obesity during childhood $(5,6)$ or adolescence $(7,8)$ promotes MS. Metabolic changes associated with obesity disrupt lipoproteins and their content and are often dubbed dyslipidemia, i.e. elevated total cholesterol (tChol), low density lipoprotein cholesterol (LDL) and triglycerides, and decreased high density lipoprotein cholesterol (HDL). This constellation has been associated with poor outcome of MS (9-14). It has been further proposed that cholesterol modulates the immune system and that hypercholesterolemia drives a proinflammatory response (15). However, cholesterol is also an indispensable component of the CNS: it is a component of cellular membranes and of myelin (16), is required for synapse and dendrite formation (17) and for axonal guidance (18). Thus the role of cholesterol metabolism during MS is largely debated and the underlying mechanisms remain unclear.

Initial studies have looked at the relationship between lipid metabolism and neuroinflammation by assessing the effect of statins, an enzymatic inhibitor of the 3-hydroxy-3-methylglutaryl coenzyme A reductase, the first line drug to lower cholesterol synthesis used in cardiovascular prevention. While statins dampen the severity of the MS mouse model, the experimental autoimmune encephalomyelitis (EAE), it has been later suggested that the beneficial effect of statins, could be independent of their cholesterol-lowering effects and related to their immunomodulatory activities similar to the ones observed with approved MS medications (4, 19-21). Moreover, the beneficial effect of statins remains controversial as studies have led to 
contradictory results in MS and their different subsets, relapsing remitting MS (RRMS) versus secondary progressive MS (SPMS) (22). Hypocholesterolemia could on the other hand be deleterious for myelin formation and for myelin repair, a process that is beneficial in MS disease, at least in animal models such as in EAE and in the cuprizone model targeting more specifically demyelination (23-25). Furthermore, sex hormones could modify immunomodulatory lipoprotein functions and the impact of lipoprotein may differ between female and male mice (26). Indeed in mouse models, LDLr deficiency was shown to attenuate EAE disease severity only in female mice through the induction of apolipoprotein E (Apo E) (27) and it has been proposed that EAE disease is less severe in ApoE deficient mice (28). However, other studies have in the contrary shown that EAE disease was more severe in ApoE deficient mice by promoting $\mathrm{BBB}$ permeability $(29,30)$. Of note in the ApoE studies, the sexspecific effects of the animals could contribute to the different results observed $(26,31)$. While a role of ApoE in EAE and MS had initially been suggested, the specific association between ApoE and a higher susceptibility risk for MS was not confirmed in a large scale genome-wide association study (GWAS) (32). Overall it is not clear a) whether the elevated circulating cholesterol levels observed in MS patients promotes inflammation; b) if elevated cholesterol levels could be needed for tissue repair in the CNS; c) and whether cholesterol lowering drugs can be considered as a treatment in MS. Moreover, few studies have determined the exact contribution of altered lipid profiles and especially cholesterol in the progression of the disease. Thus, studies investigating the role of cholesterol in demyelinating diseases should be carried out to clarify the role of cholesterol in MS.

Recently, one of the greatest advances in clinical lipidology has been the development of monoclonal antibodies targeting the Proprotein convertase subtilisin/kexin type 9 (PCSK9). Discovered in 2003, PCSK9 is a serine protease that promotes the intralysosomal degradation of the LDL receptor, resulting in reduced hepatic LDL uptake and increased plasma LDL 
concentrations. Monoclonal anti-PCSK9 neutralizing antibodies are currently used for a potent reduction of LDLc levels by $50-60 \%$ and are indicated for patients with familial hypercholesterolemia or those who are statin intolerant who need cardiovascular prevention. Their putative role has been explored in neurodegenerative disorders, in particular in Alzheimer disease (AD), where cholesterol pathways might be also involved (33). However, the contribution of PCSK9 in AD pathogenesis is controversial (34). Moreover, the role of PCSK9 has not been studied during neuroinflammation, nor on EAE or during MS.

In the present work, we show that increasing or conversely reducing blood cholesterol does not alter the peripheral adaptive immune responses during the acute phase of EAE disease. This study provides new evidence that the sole lowering of circulating cholesterol might not be sufficient to target neuroinflammation and further suggests that the beneficial effects of lowering-cholesterol drugs like statins treatment in EAE are associated with non-cholesterolrelated processes rather than with the specific decrease of circulating cholesterol.

\section{Methods}

$\underline{\text { Animals }}$

C57BL/6J and LDLr ${ }^{-/-}$(C57BL/6J background, Jackson Laboratory, stock number: 002207) mice were bred in the animal facility at Lausanne University Hospital under specific-pathogen free conditions. The animals had access to food and tap water ad libitum with a constant 12-h light/dark cycle. All mice were aged between 8 and 10 weeks. All procedures and methods were performed in accordance with guidelines from the Cantonal Veterinary Service of canton of Vaud, Switzerland (authorization \#VD3393a).

\section{$\underline{\text { EAE induction and clinical evaluation }}$}


119 For induction of EAE, mice were immunized with $100 \mu \mathrm{g}$ myelin oligodendrocyte glycoprotein (MOG) peptide 35-55 (MOG $35-55$ ) (Anawa) or PBS emulsified in complete Freund's adjuvant supplemented with $5 \mathrm{mg} / \mathrm{ml}$ Mycobacterium tuberculosis H37Ra (BD Difco). A total of $200 \mu 1$ emulsion was subcutaneously injected into four sites on the flanks of mice. At days 0 and 2 after initial peptide injections, animals received additional intravenous injection of $100 \mathrm{ng}$ pertussis toxin (Sigma Aldrich). Mice were scored daily for clinical symptoms. The EAE symptoms were assessed according to the following score: score 0 - no disease; score $0.5-$ reduced tail tonus; score 1 - limp tail; score 1.5 - impaired righting reflex; score 2 - limp tail, hind limb weakness; score 2.5 - at least one hind limb paralyzed; score 3 - both hind limbs paralyzed; score 3.5 - complete paralysis of hind limbs; score 4 - paralysis until hip; score 5 moribund or dead. Mice were euthanized if they reached a score $>3$.

\section{$\underline{\text { Antibody treatment }}$}

For lowering cholesterol experiments, mice were intraperitoneally injected with $10 \mathrm{mg} / \mathrm{kg}$ of anti-PCSK9 (proprotein convertase subtilisin/kexin type 9) (alirocumab) or PBS control one week before EAE immunization and once per week until the end of the experiments.

\section{Quantification of lipid profile}

Blood from mice were collected submandibular and serum was isolated using centrifugation. Serum lipid profiles were assessed using Roche Cobas C111 robot from the Mouse Metabolic Evaluation Facility (University of Lausanne, Switzerland) and Siemens Dimension Xpand plus from the Center of Phenogenomics (EPFL, Lausanne, Switzerland).

\section{$\underline{\text { Antigen-specific proliferative and cytokine responses }}$}


Single cell suspensions were prepared from spleens 10 days post-immunization for EAE. Cells were restimulated with $\mathrm{MOG}_{35-55}$ for $72 \mathrm{~h}$ in supplemented DMEM medium containing inactivated 10\% FCS (FBS 18, Biowest), $100 \mathrm{U} / \mathrm{mL}$ penicillin-streptomycin (BioConcept), 1 mM sodium pyruvate (Sigma), 50 M $\beta$-mercaptoethanol (Gibco), MEM non-essential amino acids (100x) (Gibco), MEM vitamins (100x) (Sigma), $200 \mathrm{mM}$ L-glutamine, folic acid 14mM (Sigma), 0.3 mM L-asparagine (Sigma), 0.7 mM L-arginine. For proliferation assays, cells were pulsed with $1 \mu \mathrm{Ci}$ of [3H]-thymidine (Hartmann Analytic) during the final $18 \mathrm{~h}$ and analysis of incorporated $[3 \mathrm{H}]$-thymidine was performed in a $\beta$-counter (Packard Top Count NXT Luminescence and Scintillation Counter). Secreted cytokines were measured after $48 \mathrm{~h}$ of culture with $\mathrm{MOG}_{35-55}$ by ELISA (Invitrogen).

\section{$\underline{\text { Isolation of immune cells }}$}

Mice were perfused through cardiac ventricle with Phosphate-buffered saline (PBS) $1 \times$. Brain and spinal cord were cut into pieces and digested $45 \mathrm{~min}$ at $37^{\circ} \mathrm{C}$ with collagenase $\mathrm{D}(2.5 \mathrm{mg} / \mathrm{ml}$; Roche) and DNAse I (1mg/ml; Roche) followed by 70\%/ 37\%. Percoll gradient (GE Healthcare) centrifugation. The cellular suspensions were washed and filtered through $40 \mu \mathrm{m}$ cell strainer and resuspended in culture medium for further analysis.

\section{$\underline{\text { Flow cytometric analysis }}$}

Single-cell suspensions in PBS $1 \times$ were stained with fixable viability dye eFluro ${ }^{\mathrm{TM}} 620$ (eBioscience). Cells were preincubated with anti-CD16/32 for 10 min to block Fc receptors and stained in FACS buffer (PBS containing 1\% BSA) with directly labeled monoclonal antibodies for $30 \mathrm{~min}$. For intracellular cytokine staining, cells were activated for $4 \mathrm{~h}$ with $50 \mathrm{ng} / \mathrm{ml}$ PMA, $1 \mu \mathrm{g} / \mathrm{ml}$ ionomycin in the presence of $10 \mathrm{mg} / \mathrm{ml}$ brefeldin A. After surface staining, cells were fixed and permeabilized using Foxp3/transcription factor staining buffer set and stained 
intracellularly with directly labeled monoclonal antibodies for $30 \mathrm{~min}$. Data were acquired on LSR II cytometer and all data were analyzed using FlowJo software. Fluorochrome-conjugated antibodies were purchased from several commercial sources indicated below. Antibodies against CD45 (30-F11) was from Biolegend; CD3 (145-2C11), CD4 (GK1.5), IL-17A, RORץT (Q31-378) (ebio17B7) and IFN- $\nu$ (XMG1.2) were from eBiosciences.

\section{$\underline{\text { Statistical analysis }}$}

Data analyses and graphs were performed using GraphPad Prism 7.0 software. P-values $<0.05$ were considered significant. Results are displayed as mean and SEM, or mean and SD, as described in the figure legends.

\section{Results}

\section{LDLr deficiency does not impact EAE disease progression}

A previous report described a protective role of LDLr deficiency in female but not in male EAE mice (27), however the nature of the observed sexual dimorphism remains unclear. Here we first evaluated female $\mathrm{LDLr}^{-/-}$mice. We confirmed that $\mathrm{LDLr}^{-/-}$mice exhibited higher blood cholesterol. Blood was collected from WT and $\mathrm{LDLr}^{-/-}$female mice before induction of EAE. $\mathrm{LDLr}^{-/-}$mice had a significant two-fold elevation of total cholesterol compared to WT mice (Fig. 1a). To elucidate whether hypercholesteremia impacts EAE severity, WT and LDLr ${ }^{-/}$ female mice were immunized with $\mathrm{MOG}_{35-55}$ peptide. We observed similar EAE disease course in both groups (Fig. 1b). The experiment was repeated in male mice to assess the sex-dependent effects of hypercholesteremia on EAE severity. We observed that male $\mathrm{LDLr}^{-/-}$mice exhibited similar EAE disease course compared to their respective WT group (Fig. 1c). In our settings, we did not observe any impact of LDLr deficiency on the development of EAE. 
Antigen-activated $\mathrm{T}$ cells are key effector cells in the pathogenesis of EAE, which are first activated in secondary lymphoid organs where they expand before migrating to the CNS.

196

Moreover, T cells are dependent on cholesterol to proliferate (35). We thus investigated the influence of LDLr deficiency on T cell responses in periphery at preclinical stage of EAE. As we did not observe sex differences in EAE disease (Fig.1, section above), we continued our analysis on female mice only as it is usually performed in EAE. WT and $\mathrm{LDLr}^{-/-}$mice were immunized with $\mathrm{MOG}_{35-55}$ emulsified in $\mathrm{CFA}$ and pertussis injections. After 8 days, splenocytes from both $\mathrm{LDLr}^{-/}$and WT mice were isolated and stimulated with $\mathrm{MOG}_{35-55}$ peptide in vitro. Proliferation and activation in response to $\mathrm{MOG}_{35-55}$ were assessed by thymidine incorporation and IL-2 secretion in the culture supernatants. No significant differences in $\mathrm{T}$ cell mitotic activities were observed between $\mathrm{LDLr}^{-/-}$and $\mathrm{WT}$ splenocytes induced with $\mathrm{MOG}_{35-55}$ and CFA (Fig. 2a) and no significant changes in IL-2 levels in the supernatant were detected (Fig. 2b). Furthermore, we explored the frequency IL17A ${ }^{+} / \mathrm{IFN}^{+}$ double producing and $\mathrm{RORT}^{+} / \mathrm{IL}_{-}-17 \mathrm{~A}^{+} \mathrm{CD} 4^{+} \mathrm{T}$ cells in activated CD4 $\mathrm{T}$ lymphocytes $\left(\mathrm{CD}^{+} \mathrm{CD}^{+} \mathrm{CD} 44^{+}\right)$after 6 days of culture with $\mathrm{MOG}_{35-55}$ by flow cytometry. We observed that both IL-17A ${ }^{+} /$INF- $\gamma^{+}$double producing CD4 lymphocytes (Fig. 2c) and RORT ${ }^{+} / \mathrm{IL}-17 \mathrm{~A}^{+}$(Fig. 2d) were equivalent in WT and $\mathrm{LDLr}^{-/}$mice. These data indicate that antigen-specific sensitization with $\mathrm{MOG}_{35-55}$ is not impaired in the absence of LDLr.

Monoclonal anti-PCSK9 neutralizing antibody decreases circulating cholesterol without alleviating EAE symptoms

As we observed that LDLr deficiency did not affect EAE disease progression, we further explored whether on the other hand, reducing circulating cholesterol would attenuate the disease as described for statin treatment. Indeed, an effector mechanism responsible for this 
statin-mediated disease amelioration could be independent of their cholesterol-lowering properties. Therefore, we investigated the effects of a more selective class of loweringcholesterol using the monoclonal antibody targeting circulating PCSK9 alirocumab, on the acute inflammatory responses and the progression of the disease. WT mice were treated either with anti-PCSK9 antibodies or control injections of PBS one week before EAE and once weekly until the end of the disease. We confirmed a significant reduction of circulating tChol, LDL and HDL in mice treated with anti-PCSK9 (Fig. 3a-3c) compared to the PBS injected group. However, despite the decrease of serum cholesterol, mice developed EAE disease with similar severity (Fig. 3d) and similar incidence (Fig. 3e). EAE is featured by infiltration of activated lymphocytes into the CNS leading to a local inflammatory response. Immune infiltrates were further assessed by flow cytometry in the CNS and no significant differences were observed between $\mathrm{CD} 45+, \mathrm{CD} 3+$ and CD4+ infiltration in anti-PCSK9 treated mice versus control (Fig. $3 f)$.

\section{Anti-PCSK9 treatment does not impair antigen-recall responses}

To further evaluate the role of lowering cholesterol levels in CNS autoimmunity, we asked whether anti-PCSK9 could alter immune cell activation in the periphery despite the absence of differences in clinical score. $\mathrm{MOG}_{35-55}$ antigen specific responses were compared between antiPCSK9 treated mice and controls. Eight days after EAE immunization, splenocytes were harvested and culture with $\mathrm{MOG}_{35-55}$ peptide in vitro. We assessed proliferation and activation in response to $\mathrm{MOG}_{35-55}$ by thymidine incorporation and IL-2 secretion in the culture supernatants. In vivo treatment with anti-PCSK9 during EAE did not alter the activation and the proliferation of peripheral $\mathrm{MOG}_{35-55}$ specific T cells (Fig. 4a), nor IL-2 secretion (Fig.4b). Moreover, the antigen-specific production of IL-17 (Fig. 4c) and IFN- $\gamma$ (Fig. 4d) in culture supernatants was not different in anti-PCSK9 treated versus control mice. Finally, the frequency 
lymphocytes $\left(\mathrm{CD}^{+} \mathrm{CD}^{+} \mathrm{CD}^{+} 4^{+}\right)$after 6 days of splenocyte culture with $\mathrm{MOG}_{35-55}$ assessed by flow cytometry was similar in anti-PCSK9 treated mice or control mice (Fig. 4e, 4f). These results indicate that anti-PCSK9 treatment does not impact the proliferation, nor polarization of autoreactive T cells during EAE.

\section{Discussion}

The relationship between MS pathogenesis and cholesterol homeostasis is largely debated.

Previous studies reported an association between elevated levels of total circulating cholesterol and their carrier lipoproteins with a worse outcome of the disease (36-39). However, the role of lowering cholesterol levels during MS is controversial: while statin treatments were initially proposed to be beneficial for EAE and MS, it has led to several disputed clinical studies (40). We here show that the sole modulation of circulating cholesterol levels is not sufficient to impact EAE using two strategies targeting circulating cholesterol: the use of $\mathrm{LDLr}^{-/-}$mice which causes a significant increase of blood cholesterol and of PCSK9 inhibitors which specifically reduces LDLr degradation and consequently lowers blood concentrations of cholesterol.

We first report that elevated circulating cholesterol levels induced by LDLr deficiency does not affect the development of EAE disease and furthermore that those results are independent of the sex of the mice. Moreover, we show that the genetic deletion of LDLr has no impact on exvivo $\mathrm{T}$ cell activation, proliferation and differentiation in response to $\mathrm{MOG}_{35-55}$. These data indicate that the reduced clearance of lipids from the circulation in $\mathrm{LDLr}^{-/-}$mice is not sufficient to substantially alter peripheral myelin-specific immune responses nor to influence EAE disease development. We propose that our observations are related to the circulating cholesterol and that they are independent of CNS cholesterol homeostasis as LDLr plays a critical role in the regulation of cholesterol metabolism outside the CNS (41). Indeed, plasma cholesterol concentration is profoundly altered in $\mathrm{LDLr}^{-/-}$mice but does not differ significantly in any 
extrahepatic organ, including the brain (42-44). In addition, LDL uptake from the BBB does not regulate CNS cholesterol (45) and furthermore cholesterol levels in the CNS rely exclusively on local de-novo synthesis (16). On the contrary, ApoE regulates cholesterol homeostasis within the CNS (16). Even though LDLr deficiency has been proposed to increase murine brain ApoE levels, it did not alter brain cholesterol levels (43, 44, 46-48). Previous studies on EAE using ApoE and LDLr deficient mice are discordant $(26-31,49)$. While both a milder and aggravated EAE have been described in ApoE deficient mice, a protective role of LDLr deficiency has been described but only in female mice and the differences observed were subtle (27). Our results do not confirm the results of this study as we show no impact of LDLr deficiency during EAE neither in female nor male mice. We however cannot exclude that other environmental factors linked to each animal facilities might explain those differences.

We then evaluated if reduction of circulating cholesterol could impact CNS autoimmunity using anti-PCSK9 antibodies, a new generation of lowering-cholesterol drug. While statins have an enzymatic inhibitory effect on cholesterol production, anti-PCSK9 monoclonal antibodies specifically reduces LDLr elimination. While we observed that anti-PCSK9 monoclonal antibodies significantly decreased the circulating cholesterol level in WT mice, the reduction of circulating cholesterol did not change the EAE clinical course nor did it have an impact on ex-vivo $\mathrm{T}$ cell activation, proliferation and differentiation in response to $\mathrm{MOG}_{35-55}$. We thus demonstrate here for the first time, that an isolated decrease of total blood cholesterol levels using a monoclonal anti-PCSK9 neutralizing antibodies does not alter the adaptive immune responses during the development of CNS autoimmunity.

Those results suggest that the effects of statins on EAE are independent of their impact on circulating cholesterol. Indeed, a study applying structural equation models proposed that the benefits of simvastatin in secondary progressive MS were probably independent of circulating cholesterol (50). Furthermore, statins could have a direct biological effect in the CNS. 
Interestingly, simvastatin, which is a small lipophilic molecule that can easily cross the BBB, has been proposed to inhibit CNS remyelination (25). This would be in line with the observation that exogenous cholesterol can enter the CNS through an impaired BBB, resulting in enhanced repair and an amelioration of the neurological phenotype in two distinct models of remyelination (23). We thus cannot exclude that lipophilic statins have an effect on cholesterol homeostasis directly in the CNS. On the contrary, monoclonal antibodies like the anti-PCSK9 antibodies, are large hydrophilic molecules that do not have the capacity to cross the BBB , especially under the conditions where the integrity of BBB is intact (51). In some pathological conditions, such as diabetes, the BBB might be compromised (52). However, even in those conditions, it is less likely that the antibodies cross the BBB (53). Even in the unlikely presence of the anti-PCSK9 monoclonal antibodies is the CNS, it has been proposed that they do not affect brain PCSK9 levels (34). These results suggest that the protective outcome of statins in EAE and possibly in MS are independent of their effect on lowering peripheral cholesterol. However, in contrast to statins whose pleiotropic effects have been reported including antiinflammatory effects and immunomodulation which are beyond the decreased circulating cholesterol, relatively little is yet known about other systemic effects of PCSK9 monoclonal antibodies. This remains to be investigated in the future.

In conclusion, we here show that enhancing or decreasing circulating cholesterol level does not have an impact on EAE disease. Interestingly, high-fat diet has been shown to exacerbate EAE disease course $(54,55)$ but a sole high-cholesterol chow was not and on the contrary could even dampened inflammation in EAE (23). We thus hypothesize that MetS and not the sole hypercholesterolemia impact neuroinflammation. In a large cohort of MS patients, MetS which comprises not only dyslipidemia, but also elevated blood pressure and type 2 diabetes was positively correlated with the severity and worse outcomes in MS (56). Thus the correction of 
the MetS and not solely the use of lipid lowering drug that mostly impact circulating cholesterol

318 should be evaluated in MS.

\section{Conclusion}

321 Our study demonstrates that circulating cholesterol does not impact the development of EAE 322 disease. It further supports the hypothesis that statin beneficial effects cannot be attributed to 323 the sole lowering serum cholesterol levels and its consequent improved hyperlipidemia, which 324 is known to be comorbidity in MS (57). Nevertheless, this does not rule out that cholesterol is 325 still an interesting target and should be further investigated especially during the recovery phase of CNS autoimmunity.

\section{Abbreviations}

MS: Multiple sclerosis, CNS: central nervous system, TG: triglycerides, tChol: total cholesterol, HDLc: high-density lipoprotein cholesterol, LDLc: low-density lipoprotein cholesterol, EAE: Experimental autoimmune encephalomyelitis, RRMS: relapsing remitting MS, PMS: secondary progressive MS, ApoE: Apolipoprotein E, PCSK9: Proprotein convertase subtilisin/kexin type 9, BBB: the blood-brain barrier, MOG: myelin oligodendrocyte glycoprotein

\section{Declaration}

\section{Ethics approval and consent to participate}

All experiments were performed in accordance with guidelines from the Cantonal Veterinary

Service of state Vaud (authorization \#VD3393a).

\section{Consent for publication}

Not applicable. 
343 Further information and requests for resources and reagents should be directed to and will be

344 fulfilled by the Lead Contact, Caroline Pot (caroline.pot-kreis@chuv.ch). This study did not

345 generate new unique reagents.

\section{Competing interests}

347 The authors declare no conflict of interest.

$348 \quad$ Funding

349 This work was supported by the Swiss National Science Foundation (PP00P3-157476 and 350 310030-192738). CP's research is supported by the Swiss Multiple Sclerosis Society and the 351 Leenaards Foundation. F.R. holds a grant from the Swiss National Science 352 Foundation (323630-183987). T.H.C.'s research is supported by grants from the Swiss National 353 Science Foundation (PZ00P3-167826), the Swiss Society of Endocrinology and Diabetes, the 354 Leenaards Foundation, and the Vontobel Foundation.

\section{Author's contributions}

356 Conceptualization, D.D., S.V., and C.P.; Methodology, D.D., S.V.; Investigation, D.D., S.V., 357 Y.Y., F.R.; J.R; B.P; Writing - Original Draft, D.D., S.V., and C.P.; Writing - Review \& 358 Editing, D.D., S.V., T.H.C., C.P.; Resources, C.P.; Funding Acquisition, C.P.

\section{Acknowledgements}

360 We thank the Service of Immunology and Allergy, Department of Medicine, of the Lausanne 361 University Hospital for daily support. We thank Mathieu Canales for technical assistance. 362 Animal Facilities of the University of Lausanne are gratefully acknowledged.

\section{References}

365 1. Reich DS, Lucchinetti CF, Calabresi PA. Multiple Sclerosis. N Engl J Med. 366 2018;378(2):169-80. 
2. Waubant E, Lucas R, Mowry E, Graves J, Olsson T, Alfredsson L, et al. Environmental and genetic risk factors for MS: an integrated review. Ann Clin Transl Neurol. 2019;6(9):190522.

3. Marrie RA. Comorbidity in multiple sclerosis: implications for patient care. Nat Rev Neurol. 2017;13(6):375-82.

4. Gianfrancesco MA, Barcellos LF. Obesity and Multiple Sclerosis Susceptibility: A Review. J Neurol Neuromedicine. 2016;1(7):1-5.

5. Langer-Gould A, Brara SM, Beaber BE, Koebnick C. Childhood obesity and risk of pediatric multiple sclerosis and clinically isolated syndrome. Neurology. 2013;80(6):548-52.

6. Munger KL, Bentzen J, Laursen B, Stenager E, Koch-Henriksen N, Sørensen TI, et al. Childhood body mass index and multiple sclerosis risk: a long-term cohort study. Mult Scler. 2013;19(10):1323-9.

7. Hedström AK, Olsson T, Alfredsson L. High body mass index before age 20 is associated with increased risk for multiple sclerosis in both men and women. Mult Scler. 2012;18(9):1334-6.

8. Munger KL, Chitnis T, Ascherio A. Body size and risk of MS in two cohorts of US women. Neurology. 2009;73(19):1543-50.

9. Giubilei F, Antonini G, Di Legge S, Sormani MP, Pantano P, Antonini R, et al. Blood cholesterol and MRI activity in first clinical episode suggestive of multiple sclerosis. Acta Neurol Scand. 2002;106(2):109-12.

10. Stampanoni Bassi M, Iezzi E, Buttari F, Gilio L, Simonelli I, Carbone F, et al. Obesity worsens central inflammation and disability in multiple sclerosis. Mult Scler. 2020;26(10):1237-46.

11. Tettey P, Simpson S, Jr., Taylor B, Blizzard L, Ponsonby AL, Dwyer T, et al. An adverse lipid profile is associated with disability and progression in disability, in people with MS. Mult Scler. 2014;20(13):1737-44.

12. Weinstock-Guttman B, Zivadinov R, Horakova D, Havrdova E, Qu J, Shyh G, et al. Lipid profiles are associated with lesion formation over 24 months in interferon- $\beta$ treated patients following the first demyelinating event. $J$ Neurol Neurosurg Psychiatry. 2013;84(11):1186-91.

13. Weinstock-Guttman B, Zivadinov R, Mahfooz N, Carl E, Drake A, Schneider J, et al. Serum lipid profiles are associated with disability and MRI outcomes in multiple sclerosis. J Neuroinflammation. 2011;8:127.

14. Durfinová M, Procházková L, Petrleničová D, Bystrická Z, Orešanská K, Kuračka L, et al. Cholesterol level correlate with disability score in patients with relapsing-remitting form of multiple sclerosis. Neurosci Lett. 2018;687:304-7.

15. Tall AR, Yvan-Charvet L. Cholesterol, inflammation and innate immunity. Nat Rev Immunol. 2015;15(2):104-16.

16. Orth $\mathrm{M}$, Bellosta S. Cholesterol: its regulation and role in central nervous system disorders. Cholesterol. 2012;2012:292598.

17. Goritz C, Mauch DH, Pfrieger FW. Multiple mechanisms mediate cholesterol-induced synaptogenesis in a CNS neuron. Mol Cell Neurosci. 2005;29(2):190-201.

18. de Chaves EI, Rusiñol AE, Vance DE, Campenot RB, Vance JE. Role of lipoproteins in the delivery of lipids to axons during axonal regeneration. J Biol Chem. 1997;272(49):3076673.

19. Aktas O, Waiczies S, Smorodchenko A, Dorr J, Seeger B, Prozorovski T, et al. Treatment of relapsing paralysis in experimental encephalomyelitis by targeting Th1 cells through atorvastatin. J Exp Med. 2003;197(6):725-33. 
20. Floris S, Blezer EL, Schreibelt G, Dopp E, van der Pol SM, Schadee-Eestermans IL, et al. Blood-brain barrier permeability and monocyte infiltration in experimental allergic encephalomyelitis: a quantitative MRI study. Brain. 2004;127(Pt 3):616-27.

21. Paintlia AS, Paintlia MK, Khan M, Vollmer T, Singh AK, Singh I. HMG-CoA reductase inhibitor augments survival and differentiation of oligodendrocyte progenitors in animal model of multiple sclerosis. Faseb j. 2005;19(11):1407-21.

22. Pihl-Jensen G, Tsakiri A, Frederiksen JL. Statin treatment in multiple sclerosis: a systematic review and meta-analysis. CNS Drugs. 2015;29(4):277-91.

23. Berghoff SA, Gerndt N, Winchenbach J, Stumpf SK, Hosang L, Odoardi F, et al. Dietary cholesterol promotes repair of demyelinated lesions in the adult brain. Nat Commun. 2017;8:14241.

24. Klopfleisch S, Merkler D, Schmitz M, Kloppner S, Schedensack M, Jeserich G, et al. Negative impact of statins on oligodendrocytes and myelin formation in vitro and in vivo. $\mathbf{J}$ Neurosci. 2008;28(50):13609-14.

25. Miron VE, Zehntner SP, Kuhlmann T, Ludwin SK, Owens T, Kennedy TE, et al. Statin therapy inhibits remyelination in the central nervous system. Am J Pathol. 2009;174(5):188090 .

26. Schrewe L, Lill CM, Liu T, Salmen A, Gerdes LA, Guillot-Noel L, et al. Investigation of sex-specific effects of apolipoprotein E on severity of EAE and MS. J Neuroinflammation. 2015;12:234.

27. Mailleux J, Timmermans S, Nelissen K, Vanmol J, Vanmierlo T, van Horssen J, et al. Low-Density Lipoprotein Receptor Deficiency Attenuates Neuroinflammation through the Induction of Apolipoprotein E. Front Immunol. 2017;8:1701.

28. Shin S, Walz KA, Archambault AS, Sim J, Bollman BP, Koenigsknecht-Talboo J, et al. Apolipoprotein $\mathrm{E}$ mediation of neuro-inflammation in a murine model of multiple sclerosis. $\mathrm{J}$ Neuroimmunol. 2014;271(1-2):8-17.

29. Zheng M, Wei J, Tang Y, Yang C, Wei Y, Yin X, et al. ApoE-deficient promotes bloodbrain barrier disruption in experimental autoimmune encephalomyelitis via alteration of MMP9. J Mol Neurosci. 2014;54(2):282-90.

30. Wei J, Zheng M, Liang P, Wei Y, Yin X, Tang Y, et al. Apolipoprotein E and its mimetic peptide suppress Th1 and Th17 responses in experimental autoimmune encephalomyelitis. Neurobiology of disease. 2013;56:59-65.

31. Dayger CA, Rosenberg JS, Winkler C, Foster S, Witkowski E, Benice TS, et al. Paradoxical effects of apolipoprotein $\mathrm{E}$ on cognitive function and clinical progression in mice with experimental autoimmune encephalomyelitis. Pharmacology, biochemistry, and behavior. 2013;103(4):860-8.

32. Lill CM, Liu T, Schjeide BM, Roehr JT, Akkad DA, Damotte V, et al. Closing the case of APOE in multiple sclerosis: no association with disease risk in over 29000 subjects. J Med Genet. 2012;49(9):558-62.

33. Shobab LA, Hsiung GY, Feldman HH. Cholesterol in Alzheimer's disease. Lancet Neurol. 2005;4(12):841-52.

34. Adorni MP, Ruscica M, Ferri N, Bernini F, Zimetti F. Proprotein Convertase Subtilisin/Kexin Type 9, Brain Cholesterol Homeostasis and Potential Implication for Alzheimer's Disease. Frontiers in aging neuroscience. 2019;11:120.

35. Bietz A, Zhu H, Xue M, Xu C. Cholesterol Metabolism in T Cells. Front Immunol. 2017;8:1664.

36. Blumenfeld Kan S, Staun-Ram E, Golan D, Miller A. HDL-cholesterol elevation associated with fingolimod and dimethyl fumarate therapies in multiple sclerosis. Mult Scler J Exp Transl Clin. 2019;5(4):2055217319882720. 
37. Browne RW, Jakimovski D, Ziliotto N, Kuhle J, Bernardi F, Weinstock-Guttman B, et al. High-density lipoprotein cholesterol is associated with multiple sclerosis fatigue: A fatiguemetabolism nexus? J Clin Lipidol. 2019;13(4):654-63.e1.

38. Gafson AR, Thorne T, McKechnie CIJ, Jimenez B, Nicholas R, Matthews PM. Lipoprotein markers associated with disability from multiple sclerosis. Sci Rep. 2018;8(1):17026.

39. Zhornitsky S, McKay KA, Metz LM, Teunissen CE, Rangachari M. Cholesterol and markers of cholesterol turnover in multiple sclerosis: relationship with disease outcomes. Mult Scler Relat Disord. 2016;5:53-65.

40. Bonetti PO, Lerman LO, Napoli C, Lerman A. Statin effects beyond lipid lowering--are they clinically relevant? Eur Heart J. 2003;24(3):225-48.

41. Go GW, Mani A. Low-density lipoprotein receptor (LDLR) family orchestrates cholesterol homeostasis. Yale J Biol Med. 2012;85(1):19-28.

42. Osono Y, Woollett LA, Herz J, Dietschy JM. Role of the low density lipoprotein receptor in the flux of cholesterol through the plasma and across the tissues of the mouse. J Clin Invest. 1995;95(3):1124-32.

43. Taha AY, Chen CT, Liu Z, Kim JH, Mount HT, Bazinet RP. Brainstem concentrations of cholesterol are not influenced by genetic ablation of the low-density lipoprotein receptor. Neurochem Res. 2009;34(2):311-5.

44. Elder GA, Cho JY, English DF, Franciosi S, Schmeidler J, Sosa MA, et al. Elevated plasma cholesterol does not affect brain Abeta in mice lacking the low-density lipoprotein receptor. J Neurochem. 2007;102(4):1220-31.

45. Dehouck B, Dehouck MP, Fruchart JC, Cecchelli R. Upregulation of the low density lipoprotein receptor at the blood-brain barrier: intercommunications between brain capillary endothelial cells and astrocytes. The Journal of cell biology. 1994;126(2):465-73.

46. Fryer JD, Demattos RB, McCormick LM, O'Dell MA, Spinner ML, Bales KR, et al. The low density lipoprotein receptor regulates the level of central nervous system human and murine apolipoprotein E but does not modify amyloid plaque pathology in PDAPP mice. J Biol Chem. 2005;280(27):25754-9.

47. Quan G, Xie C, Dietschy JM, Turley SD. Ontogenesis and regulation of cholesterol metabolism in the central nervous system of the mouse. Brain Res Dev Brain Res. 2003;146(12):87-98.

48. Kim J, Castellano JM, Jiang H, Basak JM, Parsadanian M, Pham V, et al. Overexpression of low-density lipoprotein receptor in the brain markedly inhibits amyloid deposition and increases extracellular A beta clearance. Neuron. 2009;64(5):632-44.

49. Karussis D, Michaelson DM, Grigoriadis N, Korezyn AD, Mizrachi-Koll R, Chapman $\mathrm{S}$, et al. Lack of apolipoprotein-E exacerbates experimental allergic encephalomyelitis. Mult Scler. 2003;9(5):476-80.

50. Eshaghi A, Kievit RA, Prados F, Sudre CH, Nicholas J, Cardoso MJ, et al. Applying causal models to explore the mechanism of action of simvastatin in progressive multiple sclerosis. Proc Natl Acad Sci U S A. 2019;116(22):11020-7.

51. Tabrizi M, Bornstein GG, Suria H. Biodistribution mechanisms of therapeutic monoclonal antibodies in health and disease. Aaps j. 2010;12(1):33-43.

52. Rom S, Zuluaga-Ramirez V, Gajghate S, Seliga A, Winfield M, Heldt NA, et al. Hyperglycemia-Driven Neuroinflammation Compromises BBB Leading to Memory Loss in Both Diabetes Mellitus (DM) Type 1 and Type 2 Mouse Models. Mol Neurobiol. 2019;56(3):1883-96.

53. Giugliano RP, Mach F, Zavitz K, Kurtz C, Im K, Kanevsky E, et al. Cognitive Function in a Randomized Trial of Evolocumab. N Engl J Med. 2017;377(7):633-43. 
513 54. Timmermans S, Bogie JF, Vanmierlo T, Lutjohann D, Stinissen P, Hellings N, et al.

514 High fat diet exacerbates neuroinflammation in an animal model of multiple sclerosis by 515 activation of the Renin Angiotensin system. J Neuroimmune Pharmacol. 2014;9(2):209-17.

516 55. Winer S, Paltser G, Chan Y, Tsui H, Engleman E, Winer D, et al. Obesity predisposes 517 to Th17 bias. Eur J Immunol. 2009;39(9):2629-35.

518 56. Petruzzo M, Reia A, Maniscalco GT, Luiso F, Lanzillo R, Russo CV, et al. The 519 Framingham cardiovascular risk score and 5-year progression of multiple sclerosis. Eur $\mathrm{J}$ 520 Neurol. 2020.

521 57. Marrie RA, Rudick R, Horwitz R, Cutter G, Tyry T, Campagnolo D, et al. Vascular 522 comorbidity is associated with more rapid disability progression in multiple sclerosis. 523 Neurology. 2010;74(13):1041-7. 
Figure Legend

\section{Figure 1. Hypercholesterolemia induced by LDLr deficiency do not exacerbate EAE}

\section{disease.}

(A) Serum level of total cholesterol (tChol) in female $\mathrm{LDLr}^{-/-}($mean $\pm \mathrm{SD} ; n=6)$ and wild-type mice (mean $\pm \mathrm{SD} ; n=12$ ) $* * * *, \mathrm{P}<0.0001 ; \mathrm{P}$ values were determined by unpaired Student's $t$ test. (B) EAE in female wild-type and $\mathrm{LDLr}^{-/-}$mice. The course of EAE is shown as clinical score (mean $\pm \mathrm{SEM} ; n=8)$. (C) EAE in male wild-type and $\mathrm{LDLr}^{-/-}$mice. The course of EAE is shown as clinical score (mean $\pm \mathrm{SEM} ; n=9$ ). Data are representative of three experiments.

\section{Figure 2. LDLr deficiency does not influence proliferation nor cytokine production} induced by a $\mathbf{T}$ cell recall response.

(A) On day 8 after immunization, splenocytes were isolated from WT and LDLr ${ }^{-/}$mice and restimulated with $\mathrm{MOG}_{35-55}$ in vitro. The proliferative response was measured by $[3 \mathrm{H}]$ thymidine incorporation $72 \mathrm{~h}$ after restimulation with different concentrations of $\mathrm{MOG}_{35-55}$ peptide or Concanavalin A (CON A) and expressed in counts per minute (CPM) (mean $\pm \mathrm{SD}$, $n=4$ ). (B) Cytokine IL-2 production in culture supernatants after $48 \mathrm{~h}$ of culture with the indicated concentration of $\mathrm{MOG}_{35-55}$ was determined by ELISA (mean $\pm \mathrm{SD}, n=4$ ). NS, not significant $\mathrm{P}$ values were determined by two-way ANOVA with Sidak's post hoc test. (C, D) Flow cytometric analysis of $\mathrm{IL}_{-17^{+}} / \mathrm{INF}-\gamma^{+}$and $\mathrm{IL}-17^{+} / \mathrm{ROR} \gamma \mathrm{T}^{+}$expression in $\mathrm{CD}^{+} / \mathrm{CD}^{+} / \mathrm{CD}_{4} 4^{+} \mathrm{T}$ cell at day 6 after restimulation of the indicated concentration of $\mathrm{MOG}_{35}-$ ${ }_{55}($ mean $\pm \mathrm{SD}, n=3-5) *, \mathrm{P}<0.05 ; * * * *, \mathrm{P}<0.0001$; $\mathrm{P}$ values were determined by unpaired Student's $t$ test. Data are representative of three experiments. 
EAE symptoms (A) Quantification of circulating total, (B) LDL and (C) HDL cholesterol of

550

551

552

553

554

555

556

557

558

559

560

561

562

563

564

565

566

567

568

569

570 anti-PCSK9 treated mice versus control group (mean $\pm \mathrm{SD}, n=3$ ). ${ }^{*}, \mathrm{P}<0.05 ; * *, \mathrm{P}<0.01 ; \mathrm{P}$ values were determined by unpaired Student's $t$ test (A, B, and C). Data are representative of two experiments. (D) Clinical scores of EAE in immunized mice treated with anti-PCSK9 or PBS control (mean \pm SEM, $n=7$ ). (E) Disease free activity between mice treated with antiPCSK9 and PBS control mice. (F) Flow cytometry analysis of the total proportion (\%) of the leukocyte (viable $\left.\mathrm{CD} 45^{+}\right)$, lymphocyte $\mathrm{T}\left(\mathrm{CD}^{+}\right)$and lymphocyte $\mathrm{T} \mathrm{CD} 4^{+}\left(\mathrm{CD}^{+}\right)$in the $\mathrm{CNS}$ 14 days after EAE immunization (mean $\pm \mathrm{SD} ; n=4$ ). Data are representative of two experiments.

Figure 4. anti-PCSK9 treatment does not show altered systemic immune responses. In vitro restimulation of splenocytes isolated from EAE immunized anti-PCSK9 treated mice versus control group with different concentrations of $\mathrm{MOG}_{35-55}$ peptide or $\mathrm{CON}$ A. (A) Proliferative response was determined by $[3 \mathrm{H}]$-thymidine integration and expressed in counts per minute $(\mathrm{CPM})($ mean $\pm \mathrm{SEM}, n=4)$. NS, not significant; $\mathrm{P}$ values were determined by twoway ANOVA with Sidak's post hoc test. (B, C, D) Cytokine production was determined by ELISA: Secretion of IL-2 (B), IL-17A (C) and IFN- $\gamma$ (D) were measured by ELISA after $48 \mathrm{~h}$ of culture with the indicated concentration of $\mathrm{MOG}_{35-55}$ (mean \pm SEM, $\left.n=3-4\right)$. (E, F) Flow cytometric analysis of the frequencies of $\mathrm{IL}-17^{+} / \mathrm{INF}-\gamma^{+}$(E) and $\mathrm{IL}-17^{+} / \mathrm{ROR} \gamma \mathrm{T}^{+}(\mathrm{F})$ expression in $\mathrm{CD}^{+} / \mathrm{CD}^{+} / \mathrm{CD} 44^{+} \mathrm{T}$ cells at day 6 after restimulation with indicated concentration of $\mathrm{MOG}_{35-55 .}($ mean $\pm \mathrm{SD}, n=4) *, \mathrm{P}<0.05 ; * * * *, \mathrm{P}<0.01$; $\mathrm{P}$ values were determined by unpaired Student's $t$ test. Data are representative of three experiments. 


\section{Figures}
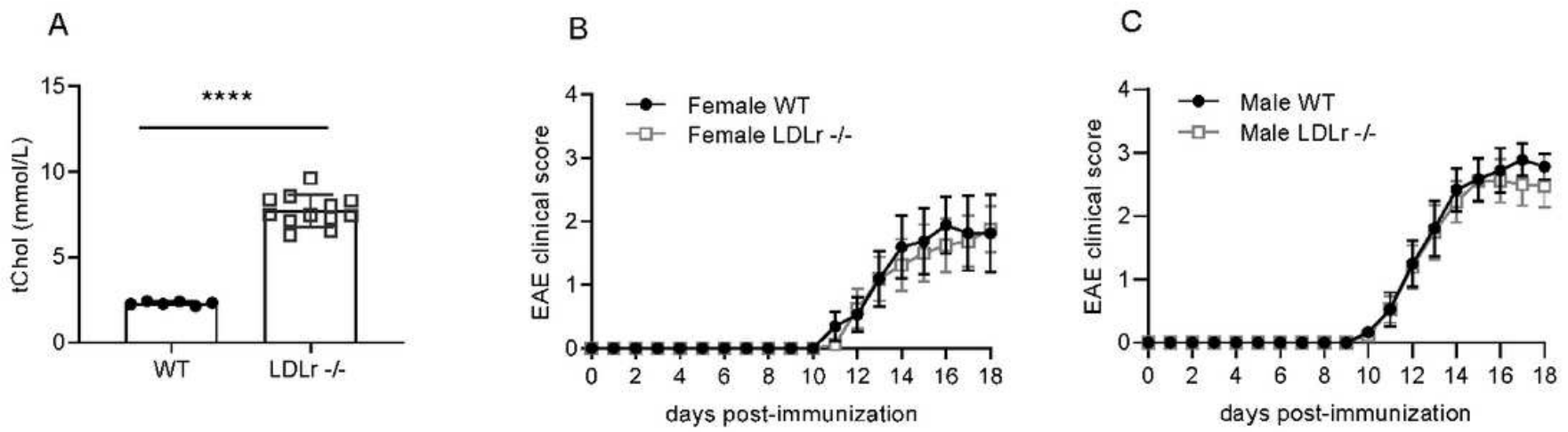

Figure 1

Hypercholesterolemia induced by LDLr deficiency do not exacerbate EAE disease. (A) Serum level of total cholesterol (tChol) in female LDLr-/- (mean $\pm \mathrm{SD} ; \mathrm{n}=6)$ and wild-type mice (mean $\pm \mathrm{SD} ; \mathrm{n}=12) \star \star \star \star \star, P<$ 0.0001; $P$ values were determined by unpaired Student's $t$ test. (B) EAE in female wild-type and LDLr-/mice. The course of EAE is shown as clinical score (mean $\pm S E M ; n=8)$. (C) EAE in male wild-type and LDLr-/- mice. The course of EAE is shown as clinical score (mean $\pm S E M ; n=9$ ). Data are representative of three experiments. 

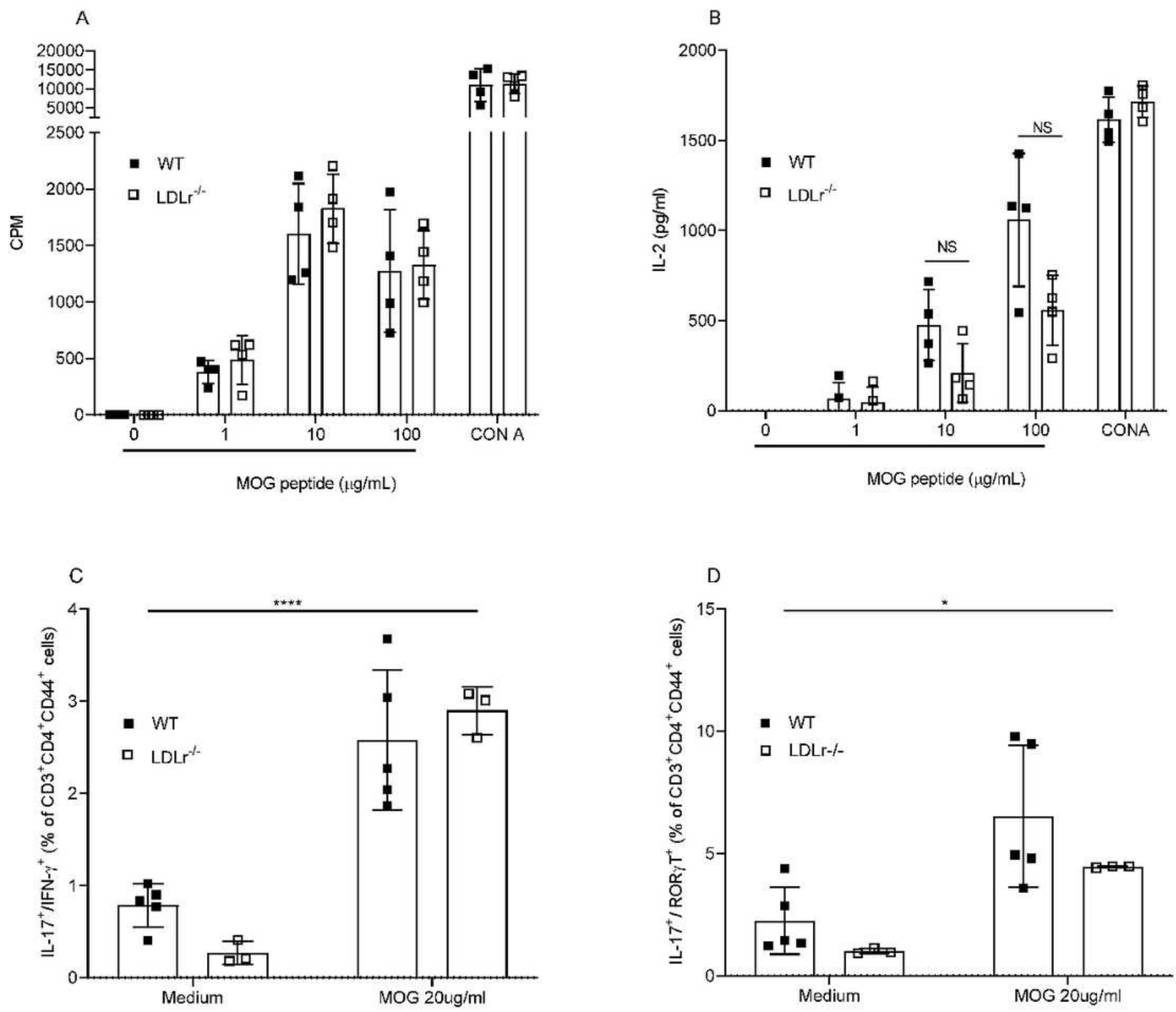

\section{Figure 2}

LDLr deficiency does not influence proliferation nor cytokine production induced by a $T$ cell recall response. (A) On day 8 after immunization, splenocytes were isolated from WT and LDLr-/- mice and restimulated with MOG35-55 in vitro. The proliferative response was measured by [3H] thymidine incorporation $72 \mathrm{~h}$ after restimulation with different concentrations of MOG35-55 peptide or Concanavalin A (CON A) and expressed in counts per minute (CPM) (mean $\pm S D, n=4$ ). (B) Cytokine IL-2 production in culture supernatants after $48 \mathrm{~h}$ of culture with the indicated concentration of MOG35-55 was determined by ELISA (mean $\pm S D, n=4$ ). NS, not significant $P$ values were determined by two-way ANOVA with Sidak's post hoc test. (C, D) Flow cytometric analysis of IL-17+/INF- $y+$ and IL-17+/RORYT+ expression in $\mathrm{CD} 3+/ \mathrm{CD} 4+/ \mathrm{CD} 44+\mathrm{T}$ cell at day 6 after restimulation of the indicated concentration of 
MOG35- 55 (mean $\pm S D, n=3-5) *, P<0.05 ; * \star \star \star, ~ P<0.0001 ; P$ values were determined by unpaired Student's t test. Data are representative of three experiments.

A
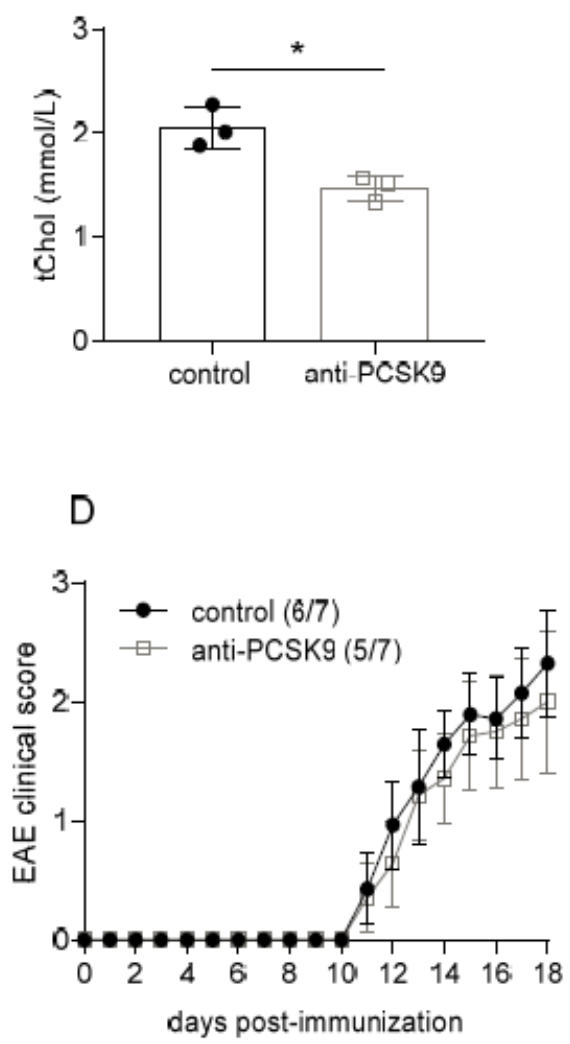

B
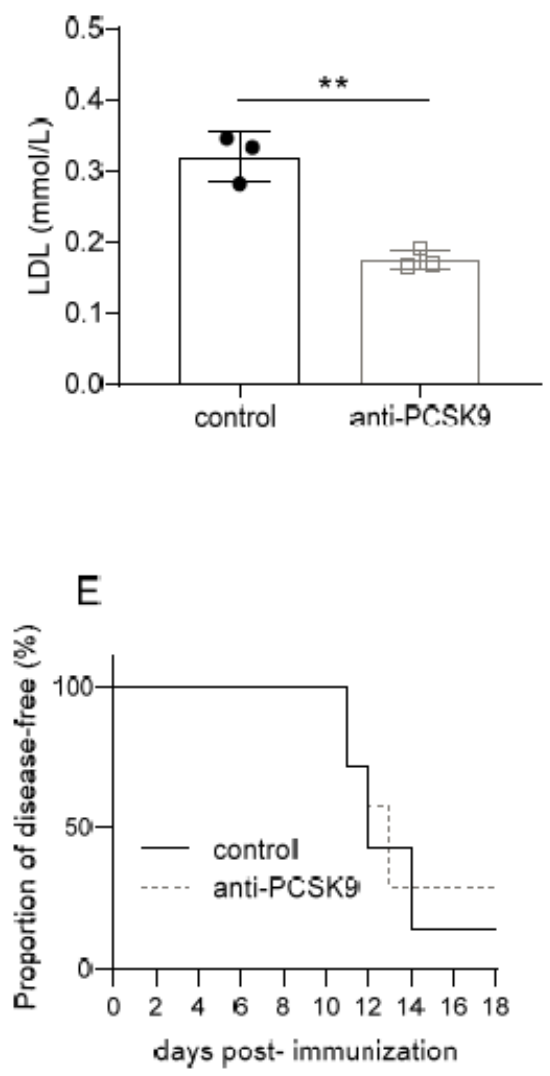

C

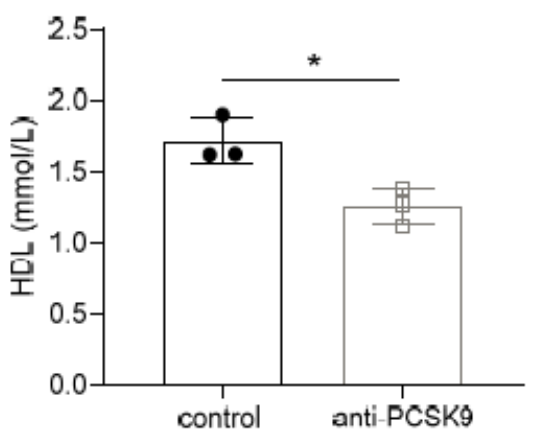

$\mathrm{F}$

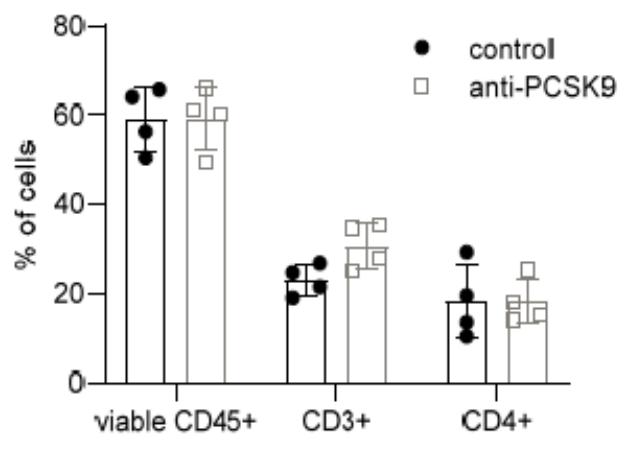

Figure 3

anti-PCSK9 decrease significantly circulating cholesterol without 548 alleviating EAE symptoms (A) Quantification of circulating total, (B) LDL and (C) HDL cholesterol of anti-PCSK9 treated mice versus control group (mean $\pm S D, n=3$ ). ${ }^{*}, P<0.05 ;{ }^{*}, \mathrm{P}<0.01 ; \mathrm{P}$ values were determined by unpaired Student's $t$ test $(A, B$, and $C)$. Data are representative of two experiments. (D) Clinical scores of EAE in immunized mice treated with anti-PCSK9 or PBS control (mean \pm SEM, $n=7$ ). (E) Disease free activity between mice treated with anti- PCSK9 and PBS control mice. (F) Flow cytometry analysis of the total proportion (\%) of the leukocyte (viable CD45+), lymphocyte T (CD3+) and lymphocyte T CD4+ (CD4+) in the CNS 14 days after $E A E$ immunization (mean $\pm S D ; n=4$ ). Data are representative of two experiments. 

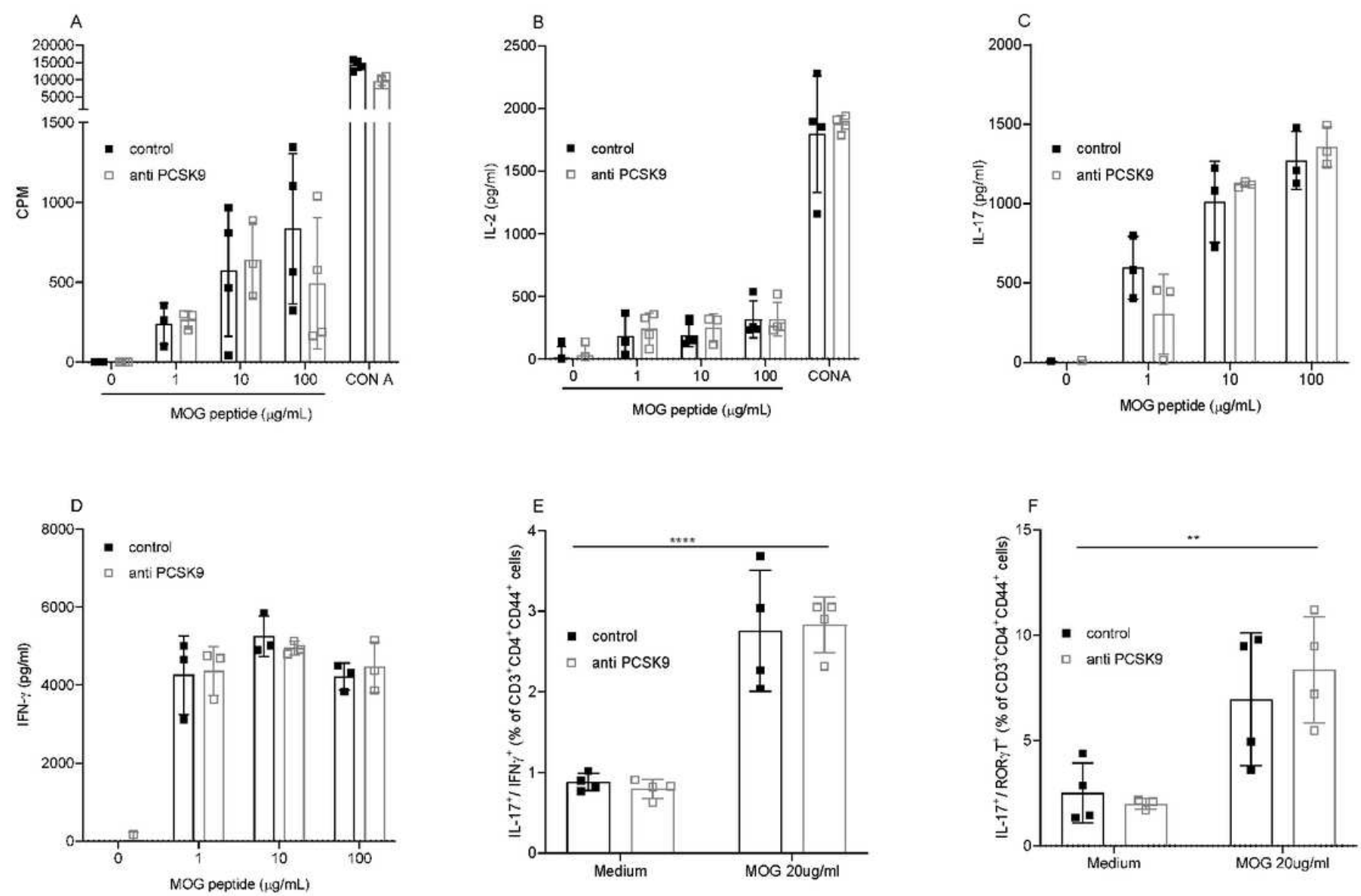

\section{Figure 4}

anti-PCSK9 treatment does not show altered systemic immune responses. In vitro restimulation of splenocytes isolated from EAE immunized anti-PCSK9 treated mice versus control group with different concentrations of MOG35-55 peptide or CON A. (A) Proliferative response was determined by [3H]thymidine integration and expressed in counts per minute (CPM) (mean $\pm S E M, n=4)$. NS, not significant; $P$ values were determined by two564 way ANOVA with Sidak's post hoc test. (B, C, D) Cytokine production was determined by ELISA: Secretion of IL-2 (B), IL-17A (C) and IFN- $\gamma$ (D) were measured by ELISA after 48 $h$ of culture with the indicated concentration of MOG35-55 (mean \pm SEM, $n=3-4)$. (E, F) Flow cytometric analysis of the frequencies of IL-17+/INF- $\gamma+(E)$ and IL-17+/RORYT+ (F) expression in CD3+/CD4+/CD44+ $T$ cells at day 6 after restimulation with indicated concentration of MOG35-55. (mean $\pm S D, n=4) *, P<$ $0.05 ; \star \star \star \star, P<0.01 ; P$ values were determined by unpaired Student's $t$ test. Data are representative of three experiments. 